\title{
PROM SSCOL - Impact of a Papillomavirus Vaccination Promotion Program in Middle School: A Study Protocol for a Randomized Controlled Trial
}

\author{
Phuong Lien TRAN ( $\square$ phuong_lien_tran@yahoo.com ) \\ Saint-Pierre University Hospital https://orcid.org/0000-0003-3283-1450 \\ Emmanuel Chirpaz \\ Centre Hospitalier de Saint Denis \\ Malik Boukerrou \\ Saint-Pierre University Hospital \\ Antoine Bertolotti \\ Saint-Pierre University Hospital
}

\section{Research Article}

Keywords: HPV vaccine, vaccination program, middle school

Posted Date: November 15th, 2021

DOI: https://doi.org/10.21203/rs.3.rs-871728/v1

License: (c) (i) This work is licensed under a Creative Commons Attribution 4.0 International License. Read Full License 


\section{Abstract}

Background: In Reunion Island, incidence and mortality for uterine cervical cancer is high, yet coverage rate for HPV vaccination is low. The main objective of the study is to evaluate, the impact of a health promotion program, promoting HPV vaccination, on the proportion of middle school girls who complete the full HPV vaccination schedule (2 or 3 doses) by the end of school year.

Methods: This study is a cluster controlled intervention study using a superiority design. A combined health promotion program will be offered, containing: information to students and parents, training of general practitioners and free school-based vaccination (in a "health bus"). Children who will benefit from this program will constitute the intervention group, and will be compared to children from another middle school who will not benefit from any program, constituting the control group.

Discussion: Our hypotheses were as follow : Clear and appropriate information for the target population as well as for their parents, will improve their knowledge about HPV vaccination, and thus increase their adherence to this vaccination ; combining information with vaccination in the school setting will reduce any material obstacles that may prevent the vaccination process; raising awareness among general practitioners will enable them to better understand the benefits and risks of HPV vaccination, and thus encourage families, who naturally trust them, to adhere to the program. Final implication would be an extension of this program in all middle schools of the Island and thus increase HPV vaccination coverage.

Trial registration: ClinicalTrials.gov ID: NCT04459221

\section{Administrative Information}

\section{Note}

the numbers in curly brackets in this protocol refer to SPIRIT checklist item numbers. The order of the items has been modified to group similar items (see http://www.equator-network.org/reporting-guidelines/spirit-2013-statement-defining-standard-protocol-items-for-clinicaltrials/).

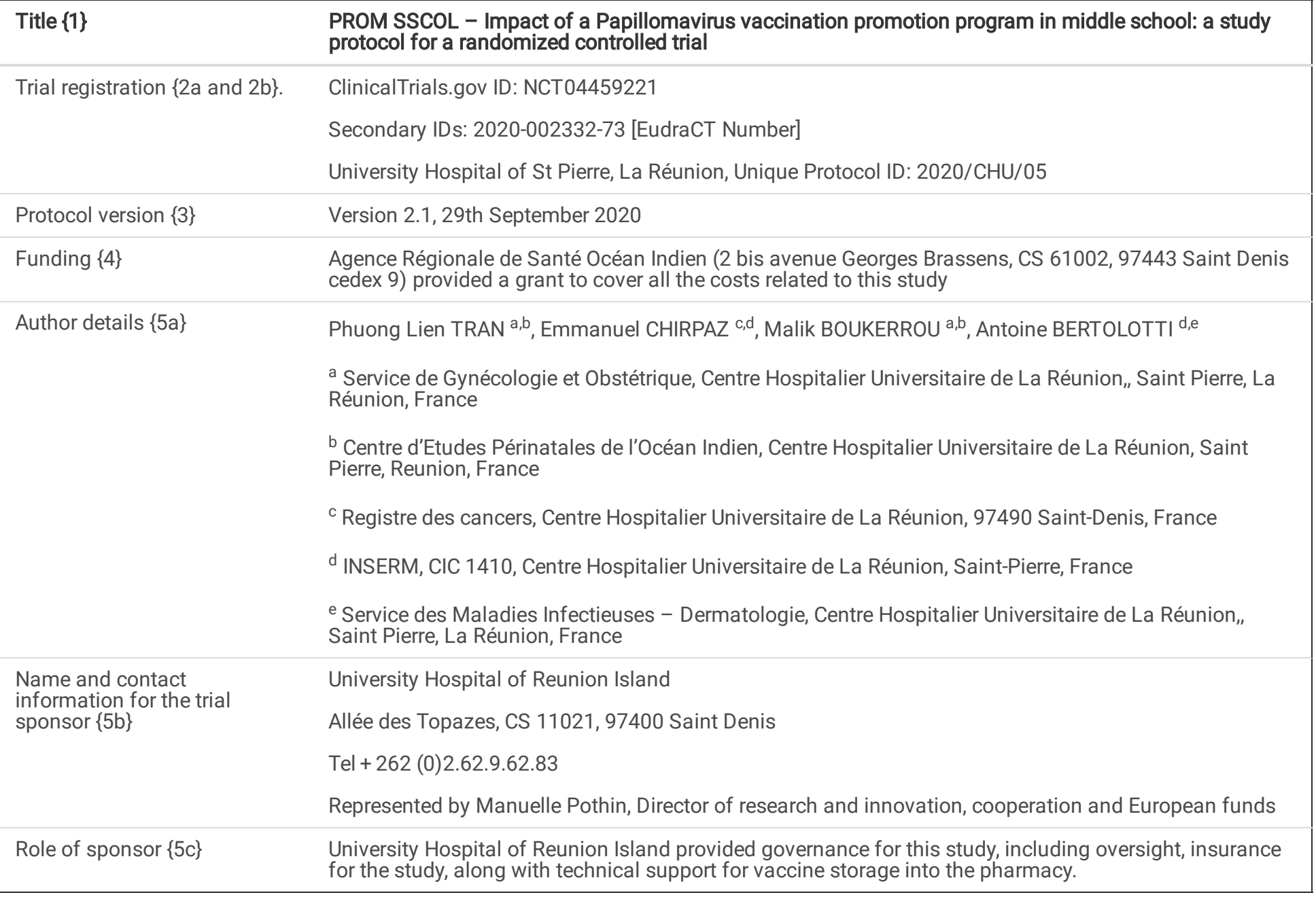




\section{Introduction}

\section{Background and rationale $\{6 a\}$}

In Reunion Island, a French territory located near the Eastern Coast of Madagascar in the Indian Ocean, uterine cervical cancer (CC) is the fourth most common cause of cancer in women, similarly to worldwide (1). However, the standardized incidence rate in 2016 was 8.8 for 100 000 women, twice higher than in Metropolitan France. The standardized mortality rate follows a similar trend: in Reunion, it accounts for 4.8 for 100000 women, whereas the Metropolitan rate was 1.7 for 100000 women $(2,3)$.

CC results from human papillomavirus (HPV) infection, which is the most common viral sexually transmitted infection. There are more than 100 types of HPV, some of which are high-risk oncogens, such as HPV 16 and 18, which are responsible for 70 to 80 percent of invasive cervical cancers (4). In Reunion Island, most frequent HPV genotypes are HPV 16, 52, 33 and 31, all contained in the nonavalent HPV vaccine (5).

Indeed, prevention of CC is mainly based on screening by cervical HPV test and on HPV vaccination, which has proven to be effective in reducing the prevalence of HPV carriage, but also in reducing the incidence of condyloma or intermediate grade dysplasia $(6,7)$. Since HPV is mainly transmitted sexually, it is important to vaccinate before the beginning of sexual life.

Because HPV infections can also lead to vulvar, vaginal, penile, anal or throat cancers, some countries (e.g., the United States, Canada, Australia, Germany, Austria, Belgium, Italy) recommend gender-neutral vaccination, in order to promote herd immunity and reduce circulation of the virus in general population $(6,8)$. In France, since December 2019, it is recommended that HPV vaccination should be offered to all children, regardless of their gender, aged 11 to 14 years (2 doses), with catch-up vaccination possible between 15 and 19 years of age for adolescents not yet vaccinated (3 doses). Before December 2019, vaccination was only recommended for girls.

High levels of vaccination coverage are obtained in countries that vaccinate in schools (9-11).

In Reunion Island, HPV vaccination coverage rate is the lowest in France, estimated at $8.1 \%$ among 16 -year-old girls in 2018 , while the national average is already low (23.7\%) (12).

This low coverage rate in Reunion Island may have several explanations. Firstly, inhabitants seem to be poorly informed about the existence of this vaccine (13). Moreover, vaccination coverage rates depend on socioeconomic level of the population. In France lower rates of HPV vaccination uptake were observed in adolescents with Universal Health Insurance Coverage (French equivalent of the US Medicaid program) compared to those not receiving such insurance (14). Reunion Island is one of the French departments with the highest rates of inhabitants under this Universal Health Insurance. Finally, not only is there a vaccination hesitancy in general, but also most specifically against HPV vaccine, among patients and also among physicians (13). Fourty one percent of Reunion inhabitants are unfavorable to vaccinations, HPV vaccine being among the most frequently cited. Among patients not vaccinated against HPV, $37 \%$ stated that the vaccine had not been suggested to them by their doctor, $7.3 \%$ were confronted with doubts expressed by their doctor concerning vaccination in general (13). However, a systematic review of 79 studies in 15 countries $(15,16)$ showed that the most important factor influencing HPV vaccination was physician recommendation. Indeed, $89.3 \%$ of the Reunion population fully trust their doctor (13). Therefore, interventions targeting health professionals and especially general practitioners appear to be paramount, especially when combined with interventions targeting the population to be vaccinated (17).

Thus, given the epidemiological situation in Reunion Island (high incidence and mortality for CC, very low coverage rate for HPV vaccination), we thought it would be interesting to study the impact of a prevention program against sexually transmitted infections, including pathologies related to HPV, with a program promoting HPV vaccination among young students in middle school.

\section{Objectives $\{7\}$}

Hypotheses were the following:

- Clear and appropriate information for the target population concerned by the vaccination (middle school students aged 9 to 17), as well as for their parents, will improve their knowledge about HPV vaccination, and thus increase their adherence to this vaccination.

- Combining information with vaccination in the school setting will improve coverage, as it will reduce any material obstacles that may prevent the vaccination process.

- Raising awareness among general practitioners will enable them to better understand the benefits and risks of HPV vaccination, and thus encourage families, who naturally trust them, to adhere to the program. 
The main objective of the study is to evaluate, in a population of middle school girls (female) in Reunion Island, the impact of a health promotion program, on the proportion of middle school girls (female) who complete the full HPV vaccination schedule (2 or 3 doses) by the end of school year.

The program conducted during school year, will combine:

- sexual health promotion (students and parents) during classes at school, at the beginning of school year,

- training of general practitioners (who practice in a perimeter of $5 \mathrm{~km}$ around the middle school) on HPV vaccination, at the beginning of school year,

- free school-based vaccination (in a "health bus"), during the academic year,

Secondary objectives in the study population at the end of school year are:

(1) To assess the impact of the combined health promotion program on the proportion of middle school girls (female) who initiated HPV vaccination (at least one dose),

(2) To assess the acceptability of the HPV vaccination program among middle school boys (male),

(3) To describe the barriers to HPV vaccination for both girls and boys,

(4) To assess the acceptability of HPV vaccination in the school setting,

(5) To assess the value of setting up a sexual health information point through a "health bus",

(6) To evaluate the satisfaction of students, parents and school workers with the measures put into place,

(7) To evaluate vaccination coverage for different mandatory vaccines, according to the current national vaccination calendar.

\section{Trial design $\{8\}$}

This study is a cluster controlled intervention study using a superiority design.

Children who will benefit from the combined health promotion program will constitute the intervention group, and will be compared to children who will not benefit from any program (as it is currently the case in all French middle schools) constituting the control group.

\section{Methods: Participants, Interventions And Outcomes}

\section{Study setting \{9\}}

This trial will concern Reunion Island, in order to investigate the particular epidemiological situation of HPV on the Island, even if the results of this study are expected to be applicable to other French regions.

The two arms of the trial will be constituted in order to:

- have in each of the two arms of the trial the most comparable populations,

- avoid any risk of contamination between the two arms and avoid having general practitioners taking care of children in both schools.

We have thus chosen to carry out a cluster trial. The two groups (intervention group and control group) will be selected from two different middle schools located in two different cities. In each of the two schools, we will randomly draw three classes in each grade level $\left(6^{\text {th }}, 7^{\text {th }}, 8^{\text {th }}\right.$ and $9^{\text {th }}$ grade) in order to have a balanced number of students in each arm (see Sample size $\{14\}$ ). Thus, 12 classes will be selected for each school.

Provided that there is a relationship between socio-economic status and vaccination coverage, it was decided to focus the study only on middle schools in priority education zone, which theoretically enroll population in which HPV vaccination coverage is the lowest. In Reunion Island, 21 middle schools are classified as priority education zone, spread over 7 cities. In agreement with the Rector and the schools directors, two schools have been designated among the above-mentioned middle schools:

- The intervention school will be Paul Hermann middle school, located in St Pierre 
- The control school will be Plateau Goyave middle school, located in St Louis

These choices are based on the schools' ability to participate in this research, their geographical location, the possibility to park the health bus inside or in the immediate surroundings of the school of the intervention group.

Health bus will be provided by a local association, Asetis, existing since 1996 and recognized as being of public interest.

\section{Eligibility criteria $\{10\}$}

Inclusion criteria

Students:

- enrolled in one of the classes randomly selected in the two middle schools designated

- affiliated to or benefiting from a social security system

- who will agree to participate in the study and whose parents or holders of parental authority will sign a free, informed and written consent

\section{Exclusion criteria}

Intervention group

Students:

- under 9 years old, or over 17 years old at inclusion

- with hypersensitivity to the active substances or to one of the excipients of the vaccine (Gardasil $9 \AA$ )

- with a permanent contraindication to vaccination

- with pregnancy or breastfeeding (based on self-reporting)

- who have already initiated HPV vaccination (complete or incomplete schedule); will be eligible to participate for collection of data but not for vaccination in the "health bus"; students with an incomplete vaccination schedule will be referred to their general practitioner to complete the missing dose(s)

$\underline{\text { Control group }}$

Students:

- under 9 years old, or over 17 years old at inclusion

Vaccinations will be performed by a junior doctor, under the supervision of a senior doctor.

\section{Who will take informed consent? $\{26 a\}$}

For the middle school assigned to the intervention group, inclusion will be achieved at the beginning of the academic year (September). For the middle school assigned to the control group, inclusion will be carried out at the end of the school year (May/June).

During school class, an envelope to bring home will be given to each student, containing:

- written information about HPV vaccination adapted to students' age

- documents outlining objectives, interventions, constraints, foreseeable risks and expected benefits of the research, and the rights of the participants in this research context,

- a consent form to participate to the study to be signed by both parents or holders of parental authority

- a socio-demographical questionnaire, including questions about HPV knowledge

Meetings with parents at the college will be scheduled at the beginning of school year, to inform parents about HPV vaccination, and explain to them this study. Consent forms will be collected during these meetings.

However, in case assemblies are forbidden by the government because of COVID-19 context, information meetings for parents will be cancelled. Thus: 
- the investigation team will call each authority holder individually by telephone, to inform them about HPV vaccination, the study, its objective, the nature of the constraints, the foreseeable risks and the expected benefits of the research. The team will also remind them of the rights of participants in research and will check the eligibility criteria. Finally, when possible, the team will collect their oral consent.

- consents will be collected remotely through the child who will bring home the consent form to be signed by his/her parents after oral consent has been obtained from them

All the documents will be returned by the student to his/her main teacher or to school nurse in a sealed envelope and then collected by the research team. Students can also return the documents directly to the research team during vaccination campaigns.

Parents or holders of parental authority will be given a period of time to consider the matter before signing the consent form. The consent of the student and the parent(s) will be required to participate. Even if the parents agree, if the student objects to the research, the student will not be included in the study. Similarly, the consent of the parent(s) will be required in addition to the consent of the underage student.

Only the signature of the parent(s) will be required, signature of the student on consent form is optional (oral consent is accepted). Parental signed consent is mandatory, oral consent alone is not sufficient.

In the Intervention group, two separate consent forms will be provided:

- an agreement for data collection

- an agreement for vaccination

It will therefore be possible to agree to data collection while refusing vaccination for students in the intervention school.

\section{Additional consent provisions for collection and use of participant data and biological specimens $\{26 \mathrm{~b}\}$}

Incorporated into the consent process for participation listed in section 'Who will take informed consent? And recruitment $\{26 a\}$ '.

\section{Interventions}

\section{Explanation for the choice of comparators $\{6 \mathrm{~b}\}$}

Refer to section 'Study setting $\{9\}$ '

- The intervention group will include 12 classes randomly selected from Paul Hermann middle school, located in St Pierre

- The control group will include 12 classes randomly selected from Plateau Goyave middle school, located in St Louis

\section{Intervention description $\{11 \mathrm{a}\}$}

Intervention group

As mentioned earlier, in case logistics initially planned (including parental and general practitioner information meeting) are not permitted due to COVID-19 health context, six interventions will be planned as follow:

(1) At the beginning of academic school year (August-September), during school class, an envelope to bring home will be given to each student, containing:

- written information adapted to students' age about HPV vaccination,

- documents outlining objectives, interventions, constraints, foreseeable risks and expected benefits of the research, and the rights of the participants in this research context,

- a consent form to participate to the study to be signed by both parents or holders of parental authority

- a socio-demographical questionnaire, including questions about HPV knowledge

(2) Parents information (October-November)

- children are supposed to bring the above-mentioned envelope to their parents 
- the investigation team will call each authority holder individually by telephone, to inform them about HPV vaccination, the study, its objective, the nature of the constraints, the foreseeable risks and the expected benefits of the research. The team will also remind them of the rights of participants in research and will check the eligibility criteria. Finally, when possible, the team will collect their oral consent.

- parents will be asked to place the documents (consent form and socio-demographic questionnaire) in an envelope and seal it before returning it to the main teacher for reasons of data confidentiality. Documents will then be collected by the investigation team.

(3) Student information about sexual Health and vaccination / Data Collection (November-December)

Children in the selected classes will be asked to bring in their health record on a specific date, along with the fulfilled sociodemographic questionnaire and signed informed consent for data collection from parents. On that day, an investigator will collect data necessary for the study in health records (especially vaccination data) for children for whom consent form was signed by the parents.

During this time, during school class, an information session about sexually transmitted diseases and vaccination will be given, lasting approximately one hour and adapted to the level of understanding (according to grade/age), in partnership with teachers.

Health records will be immediately returned to the students concerned.

(4) General practitioners information (November-December)

Eighty-eight general practitioners, working in a radius of $5 \mathrm{~km}$ around Paul Hermann middle school, will be sent an information leaflet about HPV vaccination, cervical cancer prevention, including latest literature review, and information about this study. If meetings are forbidden, general practitioners will be invited to a video call conference "Around HPV", at the beginning of school year.

(5) HPV Vaccination in the Health Bus (3 campaigns: December, February and May)

Free HPV vaccination will be offered in a "health bus" for both girls and boys. The bus will be parked in the playground, inside the school, allowing students to go there during breaks, lunchtime or after school. Vaccination periods will be predefined, so that the recommended HPV vaccination schedules can be followed.

Vaccinations will be performed by medical staff of the University Hospital of Reunion Island (junior doctor, under the supervision of a senior doctor) after informed consent to vaccination signed by either parents or holders of parental authority. The latter are invited to come along into the bus with their child.

Vaccination will be performed with nonavalent HPV vaccine. The proposed schedule is the one recommended by marketing authorization:

- children aged 9 to 14 years (girl or boy): 2-dose schedule (intramuscular), with the second dose to be administered between 5 and 13 months after the first dose.

- children aged 15 years and older (girl or boy): 3-dose schedule (intramuscular), the second dose to be administered at least 1 month after the first, the third at least 3 months after the second, all 3 doses to be administered within 1 year.

A vaccine label will be reported in the health record.

Before vaccination, absence of contraindications will be checked. In case of high fever or acute illness, the vaccination will be postponed and offered at a later date. Vaccinated persons will be monitored for at least 15 minutes after vaccination in the presence of medical staff because of adverse effects that may occur in the direct aftermath of the injection (rare anaphylactic reactions, syncope (fainting) sometimes associated with falls) or psychogenic reaction to needle injection (neurological signs such as transient blurred vision, paresthesias and tonicclonic movements of the limbs during the recovery phase).

During campaigns in February and May, $1^{\text {st }}$ dose of vaccination can be offered, though children will be asked to return to their general practitioner for subsequent doses.

The "health bus" system will be implemented as part of this study. Two students will be able to be vaccinated at the same time. A child will never be left alone with an adult inside the bus, there will always be a minimum of two adults present. Students can take advantage of this special time on the bus to receive personalized information on sexuality and obtain free condoms.

(6) Data collection in randomly selected classes (June) 
At the end of school year (June), an investigator will collect data from health record at a specific time during class. In particular, the researcher will look for the presence of de novo HPV vaccination performed by general practitioners, outside the health bus.

Signed consent of parents or holders of parental authority will be collected before any intervention in the study, i.e., before data collection and before school vaccination is carried out.

Vaccination data, even if not collected at the time of the intervention (3), can be collected either during the vaccination campaigns (5), or during the intervention (6) at the end of school year, especially for children whose parents have agreed to participate but not to be vaccinated with the "health bus". Indeed, since vaccination dates appear in the health record it will be possible afterwards to know whether the pupils were vaccinated before the interventions under study or not in order to have the vaccination rate at the very beginning of the study.

(7) Evaluation of satisfaction and barriers to vaccination (July-September)

Students, parents, member of school staff, general practitioners who volunteered, will be met, and semi-directed interview will be conducted to understand their satisfaction about the study, and barriers to vaccination.

\section{Control group}

In the control middle school, the study will take place at the end of school year (May-June), in 3 stages:

(1) Parents information about the study:

We would like to organize parents meetings to inform them, though if no parental meeting is possible due to COVID-19 pandemia, parents of children in selected classes will be sent home, an envelope containing:

- written information about HPV vaccination, and information about the study

- a socio-demographical questionnaire

- an objection form to participate to the study (data collection of health record)

Thus, if the form is returned to a teacher, investigation team will not be able to access child's health record. On the other hand, if no form is returned, it will be considered that parents do not object to data collection.

(2) Students information about sexual Health and vaccination / Data Collection

Children in the selected classes will be asked to bring in their health record on a specific date, along with the fulfilled sociodemographic questionnaire and signed informed consent for data collection from parents. On that day, an investigator will collect data necessary for the study in health records (especially vaccination data) for children for whom no objection form was returned.

During this time, during school class, an information session about sexually transmitted diseases and vaccination will be given, lasting approximately one hour and adapted to the level of understanding (according to grade/age), in partnership with teachers.

Health records will be immediately returned to the students concerned.

\section{Criteria for discontinuing or modifying allocated interventions $\{11 \mathrm{~b}\}$}

Criteria to stop any student's participation in the research will be:

- withdrawal of consent by the participant or one of the holders of parental authority.

- if a side effect occurs during an initial vaccination and contraindicated the subsequent injection(s), the vaccination schedule will be stopped, but the student will remain in the study for the continuation of the data collection.

The sponsor reserves the right to discontinue the study at any time for any reason that would be in the best interest of the participating subjects, in the event of new developments regarding HPV vaccination, or at the request of the appropriate authorities.

\section{Strategies to improve adherence to interventions $\{11 \mathrm{c}\}$}

Parents who give oral consent during first phone call in October-November, will be called a second time just before vaccination campaign in February to remind them to sign the written consent form. In order to collect as many health records as possible, investigation team will go into classrooms many times to remind students to bring parent consent form and health records.

Page $8 / 22$ 
Relevant concomitant care permitted or prohibited during the trial $\{11 \mathrm{~d}\}$

The student included in the research can participate simultaneously in another research and there will be no exclusion period at the end of the research.

There will be no compensation for participants or their parents.

No treatments or procedures are prohibited for students participating in the research.

Provisions for post-trial care $\{30\}$

All participants will continue usual follow-up and care with their general practitioner following completion of the trial. Any adverse outcomes as a result of HPV vaccination will be managed as per usual health care practice of those not involved in the study.

\section{Outcomes $\{12\}$}

The aim of this study is to compare clinical outcomes between classes from a middle school sensitized to HPV vaccination through a combined health promotion program (intervention group) and a middle school without any specific action (control group). The null hypothesis is that there is no difference in the two groups.

The primary outcome measure for this study is the proportion of middle school girls included in the study who completed the full HPV vaccine schedule ( 2 or 3 doses) by the end of the school year.

Secondary outcomes are:

(1) The proportion of middle school girls (females) included in the study who initiated the HPV vaccination (at least one dose) by the end of school year, in each of the 2 groups.

(2) - The proportion of middle school boys (males) included in the study who completed the full HPV vaccination schedule (2 or 3 doses) by the end of school year, in each of the 2 groups,

- The proportion of middle school boys (males) included in the study who initiated HPV vaccination (at least one dose) by the end of school year, in each of the 2 groups.

(3) Descriptive analysis of the causes of non-vaccination reported for students (girls and boys) who did not initiate the vaccination schedule, in each of the 2 groups.

(4) In the intervention group:

- The proportion of students who used the health bus for HPV vaccination among middle school students who completed the full HPV vaccination schedule during the school year,

- The proportion of students who used the health bus for HPV vaccination among middle school students who initiated HPV vaccination at the end of the school year.

(5) In the intervention group, the proportion of students using the health bus for sexual health information during the study period.

(6) In the intervention group, descriptive analysis of the positive and negative points reported by students, their parents, and college staff about the program.

(7) Proportion of students up to date for each type of vaccine at the end of the school year (according to the current vaccination calendar), in the study population as a whole and in each of the 2 intervention/control groups.

\section{Participant timeline $\{13\}$}

\section{Sample size $\{14\}$}

To our knowledge, there are no recent studies that have evaluated the impact of school-based vaccination on HPV vaccination coverage rates. An experimental catch-up vaccination program (diphtheria, tetanus, poliomyelitis, pertussis, measles, mumps, rubella, meningococcus $\mathrm{C}$, hepatitis B and HPV) in schools in the Vosges (Eastern France) (18) showed a participation rate ranging from $42.9 \%$ in the first year to $29 \%$ in the second year. 
The calculation of the study size is based on the expected proportion of vaccination among girls, as this is the main objective of this study and we have no data for vaccination among boys at this time.

Statistical assumptions were as follow:

- a proportion of schoolgirls who have had a complete HPV vaccination schedule at the end of school year of $6 \%$ in the control group for all students (compared to the $8.1 \%$ expected at age $16(12)$ ),

- a proportion of schoolgirls with a complete HPV vaccination schedule at the end of the school year of $20 \%$ in the intervention group,

Thus, 87 girls in each group would have to be included to demonstrate this $14 \%$ difference, at a-error $(0.05)$, and power $(1-\beta=0.80)$.

Assuming 15\% non-analyzable data (lost to follow-up, non-response), a total of 103 female students per group would have to be included. As there are roughly as many girls as boys in each class, we would have to include 206 students per group.

In order to have an equal representation of each age group, the sampling will be stratified on the grades : $6^{\text {th }}, 7^{\text {th }}, 8^{\text {th }}$, and $9^{\text {th }}$ grade. Thus, 52 students per grade and per group should be included.

If we consider that the classes contain an average of 22 students each, a minimum of 3 classes per grade should be randomly selected in each of the 2 middle schools to ensure the minimum necessary recruitment.

Thus, randomly selecting 3 classes per grade in each of the 2 middle schools will make it possible to include approximately 132 female students per group, and thus to ensure the minimum necessary recruitment.

A total of 528 are expected to be recruited (264 girls - 132 per group and 264 boys - 132 per group).

The calculation of the number of subjects required was carried out with the PASS 15 software.

\section{Recruitment $\{15\}$}

This research involve the teams of University Hospital of Reunion Island, Rectorate, the schools (selected middle schools), Asetis association, which has a "Health Bus" in which vaccination campaigns for students will be carried out.

Recruitment is supposed to be carried out during inclusion parental meetings. In case these are not possible because of the COVID-19 sanitary conditions, recruitment will take place during Student information sessions, or during vaccination campaigns (see section 'Intervention description $\left.\{11 \mathrm{a}\}^{\prime}\right)$.

For the intervention group, recruitment will started at the beginning of school year (November-December), for the control group at the end of the school year (May-June).

\section{Assignment of interventions: allocation}

\section{Sequence generation $\{16 \mathrm{a}\}$}

In priority education schools, there are classes called SEGPA : these classes, from $6^{\text {th }}$ to $9^{\text {th }}$ grade, are integrated into the middle school. They welcome young people who have significant school difficulties that cannot be resolved by academic assistance and support. There is only a small group of students (16 maximum) in each class, in order to individualize each student's progress. SEGPA classes should enable students to access at least a professional qualification.

In middle school Paul Hermann, there are 9 classes in each grade, including 2 classes of SEGPA per grade.

In middle school Plateau Goyave, there are 9 classes in $6^{\text {th }}$ grade and $9^{\text {th }}$ grade, 10 classes in $7^{\text {th }}$ and $8^{\text {th }}$ grade, including $2-3$ classes of SEGPA per grade.

In each of the two selected middle schools, 12 classes will be randomly selected in order to have a balanced number of students in each arm (see 'Sample size $\left.+\{14\}^{\prime}\right)$ :

- in order to have an equal representation of each age group, the sampling will be stratified on the grade $\left(6^{\text {th }}-7^{\text {th }}-8^{\text {th }}-9^{\text {th }}\right.$ grade),

- in order to take into account the specificities of SEGPA classes, it was decided to stratify on SEGPA classes as well. 
As main point in this comparative trial was similarity of the two groups compared, it was decided to randomly select 1 SEGPA class per grade and 2 non-SEGPA classes per grade.

Thus we will include in this trial: 256 students in Paul Hermann (intervention group) and 255 students in Plateau Goyave (control group).

\section{Concealment mechanism $\{16 \mathrm{~b}\}$}

Not applicable.

Implementation $\{16 \mathrm{c}\}$

Allocation sequence was computer-generated.

Interventions will be conducted by investigation team, with the help of teachers, and school directors.

\section{Assignment of interventions: Blinding}

\section{Who will be blinded $\{17 \mathrm{a}\}$}

For practical reasons, participants and investigators involved in study will not be blinded after study arm assignment. However, assessment of the primary and secondary outcomes by data analysts will be blinded.

\section{Procedure for unblinding if needed $\{17 \mathrm{~b}\}$}

Not applicable.

\section{Data collection and management}

\section{Plans for assessment and collection of outcomes $\{18 \mathrm{a}\}$}

See chapter 'Intervention description $\{11 a\}$ '.

Data collection concerning vaccinal status will be collected by investigation team in health records.

Acceptation of participation in the protocol implies that the investigators will make available the documents and individual data strictly necessary for the monitoring, quality control and auditing of the research, to persons having access to these documents in accordance with the legislative and regulatory provisions in force.

Source data concern all information contained in original documents, or authenticated copies of such documents, relating to clinical examinations, observations or other activities conducted in the course of research and necessary for the reconstruction and evaluation of the research. The documents in which the source data are recorded are called the source documents.

The source data will be derived from participants' health records or vaccination records as well as from questionnaires on socio-demographic data such as family, history and knowledge of HPV.

All information required by the protocol must be recorded in the observation books and an explanation must be provided for any missing data. Data should be collected as they are obtained and transcribed into these notebooks in a neat and legible manner.

Data will be collected in paper format before being entered into an electronic case report form (eCRF).

\section{Plans to promote participant retention and complete follow-up $\{18 \mathrm{~b}\}$}

Not applicable.

\section{Data management $\{19\}$}

Methodology and Data Management Center was the Clinical Investigation Center of the University Hospital of Reunion Island (CIC) - Public Health and Therapeutics Division of the University Hospital of Reunion - composed of methodologists, biostatisticians and data managers. Within the framework of the research, the Methodology and Data Management Center :

- collaborates in the design of the protocol with the coordinating investigator and oversees the methodological design of the research, 
- contributes to and finalizes the observation booklet

- creates and manages the computer database dedicated to the research,

- performs the statistical analysis of the data,

- participates in the publications and other uses of the research results.

Data will be collected on a paper observation book after the consent is signed by parents or holders of parental authority and by students. Data will be collected in the form of self-questionnaires (parents and children). Data concerning vaccination status at inclusion and at the end of the study were checked by the investigation team in the health record.

Data will be collected in paper format and will be entered into an electronic CRF (Ennov Clinical®) by a clinical study technician. Data will be saved daily. A data validation plan, defined jointly by the principal investigator and the Methodology and Data Management Center, will be developed and described in detail the controls to be performed for each variable. Once data entry will be completed, the data will be checked for consistency. Inconsistencies will be reported in the CS Test module of Ennov Clinical®. The data freeze/unfreeze process will be performed according to the procedure set up in the Methodology and Data Management Center.

\section{Confidentiality \{27\}}

In accordance with the legislative provisions in force, persons having direct access to the source data will take all necessary precautions to ensure the confidentiality of information relating to the investigational medicinal products, to the research, to the persons involved and in particular to their identity and to the results obtained. These persons, as well as the investigators themselves, are subject to professional secrecy.

During the research or at its conclusion, the data collected on the persons who lend themselves to it and transmitted to the promotor by the investigators (or any other specialized participants) will be made anonymous. Under no circumstances should the names or addresses of the individuals concerned be made public.

Participant coding procedures: only the first letter of the subject's name and surname will be recorded, along with a coded number specific to the research indicating the order of inclusion of the subjects.

The sponsor will ensure that each person who participates in the research has given written consent for access to individual data concerning him/her and strictly necessary for the quality control of the research.

The creation of a correspondence table between the identity of the participants and the allocated inclusion number will be necessary to associate the data of the 2 nd and possibly 3rd vaccine dose to the participants concerned.

This correspondence table will constitute a separate file from the analyzed database and will respect the security procedures in force.

Plans for collection, laboratory evaluation and storage of biological specimens for genetic or molecular analysis in this trial/future use $\{33\}$

There will be no specimens collected during this study

University Hospital of Reunion Island will be in charge of purchasing the vaccine doses and providing them, via the central pharmacy, to ASETIS health bus in the intervention college.

There will be no change in product packaging which was identical to the packaging at the time of purchase: $0.5 \mathrm{ml}$ glass pre-filled syringe with needles.

Labelling (in accordance with current regulations and good clinical practice) referring to use in clinical research will be carried out on the boxes of vaccines intended for the study.

The products will be brought by a staff member of ASETIS association on the days of school vaccination.

The products will be transported, respecting conditions of conservation of the vaccine (kept between $2^{\circ} \mathrm{C}$ and $8^{\circ} \mathrm{C}$, protected from light).

Expiration date will be checked before any injection.

Accounting and traceability of the doses given will be carried out by the doctors administrating vaccination.

Page $12 / 22$ 
The "Health Bus" has the capacity to store the vaccine doses in the appropriate conditions, in a fridge.

The products will be stored at the central pharmacy in University Hospital of Reunion Island and in the "Health Bus" on vaccination days.

The unused doses during the first school vaccination campaign (1st dose) will be returned to the Hospital central pharmacy to be used during the following campaigns.

At the end of vaccination campaigns, the unused and/or expired doses will be destroyed according to the regulations in force.

\section{Statistical methods}

\section{Statistical methods for primary and secondary outcomes $\{20 a\}$}

For descriptive analyses, qualitative variables will be described in terms of numbers and percentages with their $95 \%$ confidence intervals, quantitative variables will be expressed in terms of mean, standard deviation and $95 \%$ confidence interval, or in terms of median and interquartile range (25th and 75 th percentiles).

Comparability of groups at inclusion will be checked:

-bivariate comparisons of categorical variables will be performed by the $\mathrm{Chi}^{2}$ test or Fisher's exact test, depending on the conditions of application.

-Bivariate comparisons of means will be performed by the Student's t test or the Mann and Whitney test, depending on the conditions of application.

\section{Analysis of the primary outcome:}

The proportion of schoolgirls who will have completed the full HPV vaccination regimen at the end of the school year will be compared between the 2 groups (intervention/control) by the $\mathrm{Chi}^{2}$ test or Fisher's exact test, according to validity conditions.

\section{Secondary outcomes analysis:}

(1) The proportion of schoolgirls who initiated HPV vaccination (1 dose) by the end of school year will be compared between the 2 groups (intervention/control) by the $\mathrm{Chi}^{2}$ test or Fisher's exact test according to validity conditions.

(2) - The proportion of boys who will have completed the full vaccination schedule at the end of school year will be compared between the 2 groups (intervention/control) by the $\mathrm{Chi}^{2}$ test or Fisher's exact test, according to validity conditions.

- The proportion of boys who will have initiated the vaccination scheme at the end of the school year will be compared between the 2 groups (intervention/control) by the Chi2 test or Fisher's exact test, depending on the conditions of validity.

(3) The analysis of barriers to vaccination will consist of :

- Describing the causes of non-vaccination reported for students who did not initiate the vaccination schedule. Analyses will be performed for girls and boys separately

- to compare socio-demographic data, medical history, and health care utilization data between students who initiated HPV vaccination at the end of the school year and those who did not, in the intervention group. Bivariate comparisons of percentages will be performed by the Chi2 test or Fisher's exact test depending on validity conditions. For continuous variables, comparisons will be made using the Student's t test or the Mann Whitney $U$ test, depending on the conditions of validity. A multivariate analysis by logistic regression will be carried out in order to take into account confounding phenomena: the variable to be explained will be the fact of having initiated vaccination at the end of the school year, the explanatory variables entered in the model will be the variables for which the significance threshold in bi-variate analysis will be less than or equal to 0.20 .

(4) In the intervention group, will be determined:

- among students who initiated HPV vaccination at the end of school year, the proportion of students who used the health bus to initiate this vaccination,

- among students who completed full vaccination schedule at the end of school year, the proportion who completed all injections on the health bus.

Page 13/22 
(5) In the intervention group, the proportion of students who used the health bus for sexual health information.

(6) In the intervention group, descriptive analysis of the positive and negative points reported by students, their parents and school staff about this program.

(7) Proportion of students up to date for each type of vaccine (according to current vaccination calendar) at the end of school year, in the entire study population as well as in each of the two groups (intervention and control), and comparison of these proportions between the two groups by the $\mathrm{Chi}^{2}$ test or Fisher's exact test, according to validity conditions.

Analyses comparing control group to intervention group will all be performed on an intention-to-treat basis.

All hypotheses will be tested with bilateral tests and alpha risk of 0.05 , and confidence interval calculated at $95 \%$. Analyses will be performed using SAS 9.4 software (SAS Institute Cary NC).

\section{Interim analyses $\{21 \mathrm{~b}\}$}

There will be no interim analysis undertaken.

\section{Methods for additional analyses (e.g. subgroup analyses) $\{20 \mathrm{~b}\}$}

See 'Statistical methods for primary and secondary outcomes $\{20 \mathrm{a}\}$ '

Methods in analysis to handle protocol non-adherence and any statistical methods to handle missing data $\{20 \mathrm{c}\}$

We do not plan to use statistical methods to address missing data.

\section{Plans to give access to the full protocol, participant level-data and statistical code $\{31 \mathrm{c}\}$}

The full protocol or collected data will not be granted to the public. Participants will be provided with a lay summary of the research findings after completion of the study.

\section{Oversight and monitoring}

\section{Composition of the coordinating centre and trial steering committee $\{5 \mathrm{~d}\}$}

The scientific advisory board of the research will be composed of :

- the principal investigator,

- the methodologist of the Clinical Investigation Centre (CIC) of the University Hospital of La Réunion,

- the statistician,

- the project manager.

The Scientific Board will meet before the start of the research and at least twice a year until the end of the research.

Its mission is to make all important decisions concerning the proper conduct of the research and compliance with the protocol. It verifies the respect of ethics. It informs the Project Manager about possible problems and available results. It decides on any relevant modification of the protocol necessary for the continuation of the research, including:

- measures to facilitate recruitment into the research,

- possible modifications of the protocol,

- discussion of the results and the strategy for publication of these results.

At the end of the meeting, the president of the Scientific Board must inform the sponsor of the decisions taken. Decisions concerning a major amendment or a budget modification must be approved by the sponsor.

\section{Composition of the data monitoring committee, its role and reporting structure $\{21 \mathrm{a}\}$}

Nonavalent HPV vaccine (Gardasil9 9 ) has marketing authorization and is used in research in accordance with its intended indication. 
It will be administered in the presence of medical staff.

The expected serious and non-serious adverse events $(\mathrm{AE})$ are the same as those listed in the product's summary of product characteristics. Vaccination of boys has just been recommended since December 2019.

In view of these elements, the sponsor and the pharmacovigilant physician of the study considered that it was not necessary to constitute an independent monitoring committee for this study.

\section{Adverse event reporting and harms $\{22\}$}

The adverse events expected in the framework of the protocol are:

- Events related to the experimental management and described in the reference document (summary of product characteristics of nonavalent HPV vaccine Gardasil9®)

- Events related to the research: stress caused by vaccination

Any adverse event not included in the list of expected events or in the latest version of the reference document is qualified as unexpected.

The investigator is responsible for the collection of adverse events that occur between the collection of signed consent form and the end of student's participation.

The investigator reports adverse events of the participants included in the experimental group in the observation booklet and child's medical record. These adverse events are sought at each visit (vaccination campaigns and end of study visit) by questioning the participant and/or his/her parents. If necessary, general practitioner may be consulted.

The investigator assesses each adverse event in terms of its seriousness. The investigator must notify the Safety Unit of Bordeaux University Hospital by fax/email, without delay after becoming aware of the event.

If the investigator becomes aware of a serious adverse event (SAE), which he/she suspects to be causally related to the research, occurring after the end of the clinical trial in a participant he/she has vaccinated, he/she must inform the above-mentioned Safety Unit without delay.

The investigator must document the event as well as possible, and give the medical diagnosis if possible. The investigator should ensure that relevant follow-up information is communicated to the sponsor as soon as possible.

The investigator should submit, in addition to the SAE reporting form, copies of laboratory results or test reports or hospitalization reports documenting the serious adverse event, including relevant negative results, and should not omit to anonymize these documents and to record the patient number and code.

The investigator should follow the patient with the SAE until the SAE is resolved, stabilized at a level estimated acceptable by the investigator, or returned to the previous state, even if the patient has discontinued the research procedure. Additional information regarding the evolution of the event, if not mentioned in the first report, will be sent to the Safety Unit by the investigator.

All serious adverse events for which the investigator or the Safety Unit considers that a causal relationship is possible, are considered as suspected serious adverse events.

Note: In accordance with articles R1413-67 and L1413-14, the sponsor reminds that any health professional or any legal representative of a health establishment, medico-social establishment or service who observes a nosocomial infection or a serious adverse event related to any medical care, must report it to the General Director of the Regional Health Authority. This declaration is independent from the declaration of serious adverse events to the Safety Unit.

The occurrence of a pregnancy during or immediately after an investigation does not constitute an SAE. However, if a woman becomes pregnant during the course of research, the pregnancy must be notified in the same way as an SAE, as it will be subject to special monitoring until its outcome.

For this, the investigator informs the Safety Unit using the pregnancy notification form.

The investigator must follow the patient until the end of the pregnancy or its termination and notify the Safety Unit of the outcome. Any abnormality found in the fetus or child must be reported. Any voluntary interruption of pregnancy, medical interruption of pregnancy or miscarriage must be the subject of a pregnancy notification, and if a criterion of seriousness is present it must be notified as SAE.

Page 15/22 
The Safety Unit assesses whether the SAE is expected or unexpected based on the reference document (Summary of product characteristics of Gardasil $9 \circledast$ ). It reports to the competent authorities any suspected unexpected SAE occurring in France and outside the national territory and within the following deadlines:

1) In the case of an unexpected SAE resulting in death or endangerment of life, without delay from the day the sponsor becomes aware of it,

2) In the case of other unexpected SAE, no later than 15 days after the day the sponsor becomes aware of them.

The Safety Unit reports to the ANSM (French equivalence of US Food and Drug Administration) additional relevant information concerning unexpected SAE:

- In the case of a suspected unexpected SAE which has led to death or which is life-threatening, this information is notified within 8 days of the report above-mentioned in point 1)

- In other cases of suspected unexpected SAE and in the event of new facts, the relevant additional information shall be transmitted within a further period of 8 days from the deadline mentioned in point 2).

In this case of a study involving a medicinal product, the Safety Unit records all unexpected SAE in the EudraVigilance database.

The Safety Unit, as well as the sponsor, reports without delay any new event occurring during the research:

- to the ANSM,

- to the ethics Committee for the Protection of Individuals (CPP),

\section{Frequency and plans for auditing trial conduct $\{23\}$}

On the anniversary date of the research authorization, the Safety Unit prepares a safety report including:

- a list of SAE that may be related to the investigational treatment(s) of the research including expected and unexpected SAE, which occurred in the trial concerned during the period covered by the report,

- a concise and critical analysis of the safety of the participants involved in the research.

- summary tables of all SAE that have occurred in the trial concerned since the beginning of the research

This report is sent to the ANSM and the CPP within 60 days following the anniversary of the research authorization.

An audit may be carried out at any time by persons mandated by the sponsor and independent from those conducting the research. The objective is to verify the safety of the participants and the respect of their rights, the respect of the applicable regulations and the reliability of the data.

An inspection can also be carried out by a competent authority (ANSM).

Both the audit and the inspection may be applied to all stages of the research, from the development of the protocol to the publication of the results and the classification of the data used or produced in the course of the research.

Investigators agree to comply with the requirements of the sponsor for an audit and the competent authority for an inspection of the research.

A clinical researcher mandated by the sponsor regularly visits the investigating center, at the time the research is set up, once or several times during the research, depending on the rate of inclusion, and at the end of the research. During these visits, and in accordance with the riskbased monitoring plan (participant, logistics, impact, resources), the following elements will be reviewed

- informed consent,

- compliance with the research protocol and the procedures defined therein

- management of any products.

A written monitoring report will be prepared for each visit.

Plans for communicating important protocol amendments to relevant parties (e.g. trial participants, ethical committees) $\{25\}$

Page 16/22 
Any substantial modification, i.e. any modification likely to have a significant impact on the protection of individuals, on the conditions of validity and on the results of the research, on the quality and safety of the products tested, on the interpretation of the scientific documents that support the conduct of the research or on the methods of conducting the research, is the subject of a written amendment that is submitted to the sponsor.

Non-substantial modifications, i.e. those that do not have a significant impact on any aspect of the research, are communicated to the CPP for information purposes.

All modifications are validated by the sponsor and by all those involved in the research concerned by the modification, before submission to the CPP and, if necessary, to the ANSM. This validation may require the meeting of any committee formed for the research.

All modifications to the protocol must be brought to the attention of all investigators participating in the research. The investigators undertake to respect the content of the protocol.

Any modification that modifies the management of participants or the benefits, risks and constraints of the research is the subject of a new information note and a new consent form, the collection of which follows the same procedure as that described above.

\section{Dissemination plans $\{31 \mathrm{a}\}$}

The findings of this study will be published in peer-review journals. There are no terms or conditions to the funding that may impact upon publication and dissemination. Authorship will reflect the amount of time spent designing the study, collecting the data and writing the manuscript.

The analysis of the data provided by the investigating centers is carried out by the Clinical Investigation Center. This analysis leads to a written report that is submitted to the sponsor, who will forward it to the CPP and to the competent authority.

Any written or oral communication of the results of the research must receive prior approval from the coordinating investigator and, if applicable, from any committee set up for the research.

The coordinating/principal investigator undertakes to make available to the public the results of the research, both negative and inconclusive and positive.

In accordance with the law $n^{\circ} 2002-303$ of March $4^{\text {th }}, 2002$, the participants are informed, at their request, of the overall results of the research.

The management of the data is ensured by the Clinical Investigation Center. The conditions of transfer of all or part of the research database are decided by the research sponsor and are the subject of a written contract.

\section{Discussion}

This protocol is submitted almost a year after recruitment began, since sanitary COVID-19 condition was in constant change and evolution and it was difficult to know whether we could continue the process of the trial. Amendments were made and submitted to ethics committee, facing prohibition of meetings with more than 6 people. This protocol is the result of our constant adaptation to these different obstacles.

\section{Trial status}

Study protocol version $2.1,29^{\text {th }}$ September 2020.

Recruitment began in October 2020.

Concerning vaccination, recruitment was completed by June 2021.

Concerning evaluation of satisfaction of participants and evaluation of barriers to HPV vaccination, completion of recruitment is expected by October 2021.

\section{Abbreviations}




\begin{tabular}{ll} 
AE & Adverse event \\
\hline ANSM & Agence Nationale de la Sécurité du médicament - French equivalence of US Food and Drug Administration \\
\hline CC & Cervical cancer \\
\hline CIC & Clinical Investifation Center \\
\hline COVID-19 & Coronavirus \\
\hline CPP & Comité de Protection des personnes - ethics committee for the protection of individuals \\
\hline CRF & Case report form \\
\hline HPV & Human papillomavirus \\
\hline PROM SSCOL & Promotion of Sexual Health in College \\
\hline RGPD & Régime général de protection des données - general data protection regulation \\
\hline SAE & Serious adverse event \\
\hline SEGPA & Sections d'enseignement general et professionnel adapté - Adapted general and vocational education sections \\
\hline SHAM & Société Hospitalière d'assurance mutuelle - Hospital insurance company
\end{tabular}

\section{Declarations}

\section{Acknowledgements}

- Members of Medical research Unit of University Hospital of Reunion Island (DRCI - PB 350 - 97448 Saint-Pierre Cedex) : Lucie AUZANNEAU, Mélanie BEGORRE who provided substantial help in protocol submission to competent authorities, and Julie RUIZ, for data collection

- Asetis association, with its "health bus" and its prevention animators, in whom children trusted to ask for information concerning sexuality and vaccination : Maryse PICARD, Stéphanie GONTHIER, Julianna DANESSINGA, Pierre RIVIERE, Fabrice FONTAINE

- Junior Doctors Xavier GILHARD, Morgane HUMBERT, Julien SITTHISONE, Eva MONDON who vaccinated children in the health bus

\section{Authors' contributions $\{31 \mathrm{~b}\}$}

PLT is the chief investigator, she conceived the study, obtained financing, led the proposal and protocol development, EC helped in protocol development and was the lead trial methodologist. MB helped to develop the proposal ; $\mathrm{AB}$ contributed to study design and substantively revised the protocol.

All authors read and approved the final manuscript.

\section{Funding $\{4\}$}

Agence de Santé Océan Indien (2 bis avenue Georges Brassens, CS 61002, 97443 Saint Denis cedex 9) provided a grant to cover all the costs related to this study, including vaccine purchase.

The funding body did not play any role in the design of the study, collection, analysis, interpretation of data or writing the manuscript.

\section{Availability of data and materials $\{29\}$}

The following documents relating to the research are archived by the investigator in accordance with Good Clinical Practice

- for a period of 15 years following the end of the research (research involving drugs, medical devices or in vitro diagnostic medical devices or research not involving a product mentioned in article L.5311-1 of the public health code),

- The protocol and any amendments to the protocol

- Observation notebooks (copies)

- The source files of participants who have signed a consent form

- All other documents and letters related to the research

- for a period of 30 years following the end of the research 
- Original copies of signed informed consents from participants and authority holders

All of these documents are the responsibility of the investigator for the regulatory archiving period.

No movement or destruction will be made without the sponsor's approval. At the end of the regulatory retention period, the sponsor will be consulted for destruction. All data, documents and reports are subject to audit or inspection.

Within one year of the completion or termination of the research, a final report will be prepared and signed by the sponsor and the investigator. This report will be made available to the competent authority. The sponsor will transmit the results of the research to the CPP and, if necessary, to the ANSM in the form of a summary of the final report within one year of the end of the research.

The final dataset will only be able to be accessed by the research team.

\section{Ethics approval and consent to participate $\{24\}$}

The sponsor and the investigators agree that this research will be conducted in accordance with the law $n^{\circ} 2012-300$ of March $5^{\text {th }}, 2012$ relating to research involving human person, as well as in accordance with the Good Clinical Practices (version 4 of November $9^{\text {th }}, 2016$ and decision of November $24^{\text {th }}, 2006$ ) and the Declaration of Helsinki (which can be found in its integral version on the website http://www.wma.net).

The research is conducted in accordance with this protocol. Except in emergency situations requiring the implementation of specific therapeutic procedures, the investigators undertake to comply with the protocol in all respects, in particular with regard to the collection of consent and the notification and follow-up of serious adverse events.

This research has received the favorable opinion of the research ethics committee (CPP) of Ouest II of Angers and the authorization of the ANSM.

The University Hospital of Reunion Island, promotor of this research, has taken out a civil liability insurance policy with hospital insurance company SHAM ( $\left.n^{\circ} 158958\right)$ in accordance with the provisions of the public health code.

The data recorded during this research are subject to computerized processing at the University Hospital of Reunion Island, responsible for data processing in compliance with the law $n^{\circ} 78-17$ of January $6^{\text {th }}, 1978$ relating to data processing, files and freedoms modified by the law 2004-801 of August $6^{\text {th }}, 2004$, modified by the law $n^{\circ} 2018-493$ of June $20^{\text {th }}, 2018$.

This research falls within the framework of the "Reference Methodology" (RM-001) in application of the provisions of Article 54 paragraph 5 of the amended Act of January 6, 1978 relating to information, files and freedoms. This change was approved by decision of January $5^{\text {th }}, 2006$, updated on July $21^{\text {st }}, 2016$. The University Hospital of Reunion Island, responsible for data processing has signed a commitment to comply with this "Reference Methodology".

The research sponsor undertakes to carry out the research in compliance with the General Data Protection Regulation (RGPD) of $27^{\text {th }}$ April 2016 implemented on $25^{\text {th }}$ May 2018.

- This research is registered in the ANSM EUDRACT database under the $\mathrm{n}^{\circ} 2020-002332-73$

- This research is registered on the website http://clinicaltrials.gov/

Authors obtained consent to participate in the study from participants and their parents (or holders of parental authority). Written, informed consent to participate was obtained from all participants.

\section{Consent for publication $\{32\}$}

Consent forms for parents are available in French in Appendix A and B.

Competing interests $\{28\}$

None of the investigators has any financial or other conflicts of interest in the process of outcomes of this trial.

\section{References}


(1) Bray F, Ferlay J, Soerjomataram I, Siegel RL, Torre LA, Jemal A. Global cancer statistics 2018: GLOBOCAN estimates of incidence and mortality worldwide for 36 cancers in 185 countries. CA Cancer J Clin 2018 Nov;68(6):394-424.

(2) Observatoire régionale de la santé La Réunion. Les cancers du col de l'utérus à La Réunion. 2010.

(3) Registres FRANCIM. 2015; Available at: http://www.invs.sante.fr/applications/cancers/francim2015/default.htm.

(4) HAS. Dépistage et prévention du cancer du col de l'utérus. 2013.

(5) Tran PL, Zafindraibe N, Ah-You N, Fernandez C, Arrivets P, Gérardin P, et al. Human papillomavirus genotyping on Reunion Island: A crosssectional study of stored tissue samples. Eur J Obstet Gynecol Reprod Biol 2020 Sep;252:294-299.

(6) Drolet M, Benard E, Perez N, Brisson M, HPV Vaccination Impact Study Group. Population-level impact and herd effects following the introduction of human papillomavirus vaccination programmes: updated systematic review and meta-analysis. Lancet 2019 Aug 10;394(10197):497-509.

(7) Chow EPF, Tabrizi SN, Fairley CK, Wigan R, Machalek DA, Regan DG, et al. Prevalence of human papillomavirus in teenage heterosexual males following the implementation of female and male school-based vaccination in Australia: 2014-2017. Vaccine 2019 Sep 24.

(8) Reisinger KS, Block SL, Lazcano-Ponce E, Samakoses R, Esser MT, Erick J, et al. Safety and persistent immunogenicity of a quadrivalent human papillomavirus types 6, 11, 16, 18 L1 virus-like particle vaccine in preadolescents and adolescents: a randomized controlled trial. Pediatr Infect Dis J 2007 Mar;26(3):201-209.

(9) Paul P, Fabio A. Literature review of HPV vaccine delivery strategies: considerations for school- and non-school based immunization program. Vaccine 2014 Jan 9;32(3):320-326.

(10) Fregnani JH, Carvalho AL, Eluf-Neto J, Ribeiro Kde C, Kuil Lde M, da Silva TA, et al. A school-based human papillomavirus vaccination program in barretos, Brazil: final results of a demonstrative study. PLoS One 2013 Apr 24;8(4):e62647.

(11) Vanderpool RC, Breheny PJ, Tiller PA, Huckelby CA, Edwards AD, Upchurch KD, et al. Implementation and Evaluation of a School-Based Human Papillomavirus Vaccination Program in Rural Kentucky. Am J Prev Med 2015 Aug;49(2):317-323.

(12) Géodes - Santé Publique France - Couverture vaccinale pour papillomavirus 2018. 2018; Available at:

https://geodes.santepubliquefrance.fr/\#bbox=-263844,6775594,2995382,1848309\&c=indicator\&f=16\&i=cv_hpv.cv_hpv\&s=2018\&view=map1. Accessed 10/06, 2019.

(13) Observatoire régional de la santé de La Réunion. 2019; Available at: https://www.ors-oceanindien.org/IMG/pdf/orsoi_tb_cancers_reunion_2019.pdf. Accessed 10/07, 2019.

(14) Blondel C, Barret A, Pelat C, Lucas E, Fonteneau L, Lévy-Buhl D.

INFLUENCE OF SOCIOECONOMIC FACTORS ON HUMAN PAPILLOMAVIRUS VACCINE UPTAKE IN ADOLESCENT GIRLS IN FRANCE BUIIetin épidémiologique hebdomadaire - Santé publique France 2019(22-23):441.

(15) Ecollan M, Partouche H, Gilberg S.

Vaccination contre le papillomavirus. Exercer. 2018;(147):412-8. .

(16) Newman PA, Logie CH, Lacombe-Duncan A, Baiden P, Tepjan S, Rubincam C, et al. Parents' uptake of human papillomavirus vaccines for their children: a systematic review and meta-analysis of observational studies. BMJ Open 2018 Apr 20;8(4):e019206-2017-019206.

(17) Campana V, Cousin L, Terroba C, Alberti C.

INTERVENTIONS AIMING AT IMPROVING VACCINE COVERAGE OF HUMAN PAPILLOMAVIRUS VACCINE Bulletin épidémiologique hebdomadaire - Santé publique France 2019(22-23):431.

(18) ARS Grand Est 2018. Amélioration de la couverture vaccinale: une expérimentation de rattrapage vaccinal en milieu scolaire dans les Vosges. . 2018; Available at: http://www.grand-est.ars.sante.fr/amelioration-de-la-couverture-vaccinale-une-experimentation-de-rattrapagevaccinal-en-milieu. Accessed 10/09, 2019.

\section{Figures}




\section{Control} school
May $\mathrm{Y}+1$

1. Information
to parents
Parental
meeting

May-June $Y+1$

2. Information to students About HPV vaccination During school class

Data collection : - Health records

- Sociodemographical questionnaire

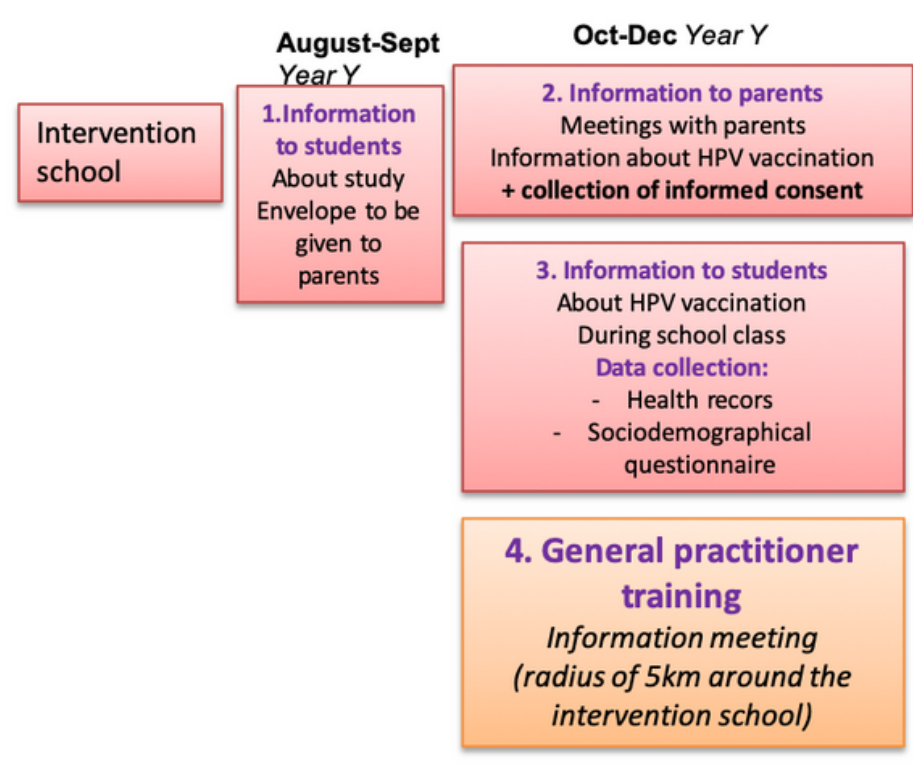

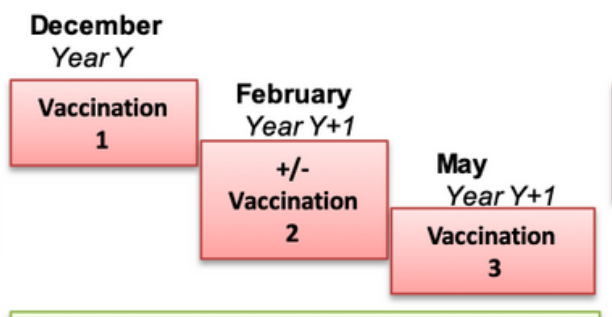

\section{HPV vaccination}

In the " health bus "

+ collection of informed consent
June Year $Y+1$

6. Data collection:

- Heath records

- Adverse events

7. Evaluation of satisfaction:

Semi-directed

interview of students

parents and school staff

Figure 1

Initial participant timeline 


\section{COVID-19}

context

School year August (Year $Y$ ) to June $($ Year $Y+1)$

\section{Control}

school
May $\mathrm{Y}+1$

$\begin{aligned} & \text { 1. Information } \\ & \text { to parents } \\ & \text { Envelope sent } \\ & \frac{\text { with written }}{\text { information }}\end{aligned}$

May-June $\gamma+1$

2. Information to students About HPV vaccination During school class

Data collection :

Health records

- Sociodemographical questionnaire
Intervention school

\begin{tabular}{l} 
August-Sept \\
Yeary \\
\hline 1.Information \\
to students \\
About study \\
Envelope to be \\
given to \\
parents \\
\hline
\end{tabular}

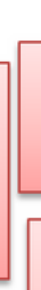

\section{December}

Year $Y$

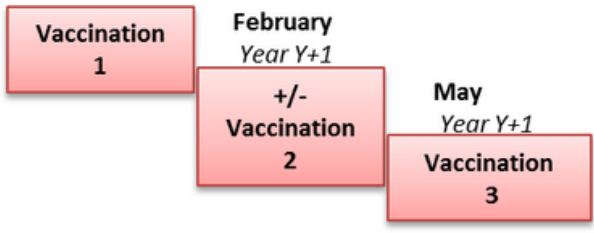

5. HPV vaccination

In the "health bus »

+ collection of written informed consent
June Year $\gamma+1$

6. Data collection:

- Heath records

- Adverse events

July-Sept Year $Y+1$

7. Evaluation of satisfaction:

Semi-directed interview of students parents and school staff

\section{Figure 2}

Participant timeline in the COVID-19 context, in case of meeting restriction

\section{Supplementary Files}

This is a list of supplementary files associated with this preprint. Click to download.

- PROMSSCOLNICEparentsintervention1.1du29.06.2020.pdf

- renamedc7b3e.pdf 